\title{
Biomarkers in heart failure: from the bedside back to biology
}

\author{
Yasmin Rustamova ${ }^{1}$, Dan Dobreanu ${ }^{2 *}$ \\ 1. Azerbaijan Medical University, Dept. of Internal Medicine 2, Baku, Azerbaijan \\ 2. University of Medicine and Pharmacy, Physiology Dept., Tîrgu Mures, Romania
}

Received: 12 $2^{\text {th }}$ July 2018; Accepted: $18^{\text {th }}$ July 2018; Published: $18^{\text {th }}$ July 2018

Through increasing survivors after myocardial infarction and oncological patients, heart failure (HF) has become the leading cause of morbidity and mortality all over the world. Treatment improvement for most cardiovascular diseases has led to a reduction in early mortality at acute events but also, by impaired heart pump function, to an increase in the number of patients with HF. As a result, HF became the price paid for the success of treatment for acute cardiovascular disease, and is now the most common ,final common pathway" to cardiovascular death and thus an important public health problem.

\section{Changing the paradigm: from broken heart to neurohumoral disorder}

According to guidelines, HF is "a clinical syndrome characterized by typical symptoms that may be accompanied by signs caused by a structural and/or functional cardiac abnormality, resulting in a reduced cardiac output and/or elevated intracardiac pressures". (1)
The essence of this definition is that HF is as a consequence of a damaged pump, and the primary objective of the evaluation becomes the demonstration of abnormality causing systolic and / or diastolic ventricular dysfunction mainly through imaging methods.

On the other hand, underfilling of the arteries and venous congestion in HF initiate a neurohumoral response in order to minimize hemodynamic abnormalities caused by failure of the cardiac pump. There are three major features of the neurohormonal response in HF. First are short-term functional signals such as the renin-angiotensin-aldosterone system and sympathetic nervous system that increase cardiac performance, constrict blood vessels and inhibit water excretion by the kidneys. This response provides mechanisms that adapt to short-term challenges, but when sustained in chronic HF can lead long-term maladaptative consequences. A second feature is mediated by proliferative (transcriptional) signaling, changes in gene expression that modify myocardial cells and the

*Corresponding author: Dan Dobreanu, University of Medicine and Pharmacy, Physiology Dept., Tîrgu Mures, Romania. E-mail: dan.dobreanu@umftgm.ro 
structure of the heart. A third type of response is proinflammatory, which may be directed to the removal of an infectious agent ("anti-other"), but which in HF may persist, causing maladaptive changes ("anti-self"). (2)

For this reason, HF is now regarded mainly as a complex neuroendocrine disorder and not as a mechanical pump dysfunction. Practical implications are major. Beyond the „mechanical” interventions for pump function (valvulopathy correction, myocardial revascularization), the only pharmacological therapies with prognostic benefits are those that directly address the reduction of inappropriate neuroendocrine activation. On the other hand, assessment of the intensity of the neurohumoral response may have prognostic significance, more important than the severity of alteration of the pump function.

\section{Biomarkers in HF: what, why?}

According to the World Health Organization, a biomarker is "any substance, structure, or process that can be measured in the body or its products and influences or predicts the incidence of outcome or disease". (3) In the clinical practice, biomarkers acquired from blood and urine specimens are used most frequently, although this definition also includes physiological features, clinical signs and biopsies results.

The neuroendocrine response in HF may cause changes in the levels of some blood parameters, which can serve as biomarkers. These biomarkers could be divided into several categories according to their involvement in different pathways of neurohumoral activation. $(4,5)$

Neurohormones (Norepinephrine, Renin, Angiotensin II, Aldosterone, Arginine-vasopressin, Endothelin) are a prompt expression of the neuroendocrine activation process in HF, even for the patients with asymptomatic left ventricular dysfunction. However, the complexity of assays for neurohormones makes them impractical as a biomarker of clinical interest in HF.
Markers of Extracellular Matrix Remodeling (Matrix metalloproteinases, Tissue inhibitors of metalloproteinases, Collagen propeptides). Both cardiomyocytes and extracellular matrix are affected in the process of heart remodeling, which is crucial for the development and progression of HF. Assessment of some enzymes degrade fibrillar collagen or collagen fragments in the blood can be used to evaluate remodeling, possibly providing prognostic information.

Inflammatory Mediators (C-reactive protein, Tumor necrosis factor $\alpha$, Fas /APO-1, Interleukins 1, 6, and 18, Galectin-3); Inflammatory response is initiated by tissue injury in order to repair this injury.

In time, this compensatory reaction has a negative effect on the structural and functional ability of the heart. As a result, the detection of the elevated level of proinflammatory cytokines, cell adhesion molecules, and chemokines in the blood could be appropriate not only in the risk assessment but also in the understanding of pathogenetic components involved in HF.

Markers of Oxidative Stress (Oxidized LDL, Myeloperoxidase, Urinary biopyrrins, Urinary and plasma isoprostanes, Plasma malondialdehyde). In the development of HF the lack of balance between reactive oxygen species (superoxide anion, hydrogen peroxide, hydroxyl radical) and endogenous antioxidant protection tools leads to oxidative stress. This can exert deleterious effects in the pathogenesis and progression of HF by damage of cellular proteins and myocyte apoptosis and necrosis. The direct measurement of reactive oxygen species in humans is challenging, although the indirect markers of oxydative stress could be used.

Markers of Myocyte injury (Cardiacspecific troponins I and T, Myosin light-chain kinase I, Heart-type fatty-acid protein, Creatine kinase MB fraction). In $\mathrm{HF}$ inflammation, oxidative stress, and neurohormonal activation cause cardiomyocyte injury and cardiomyocyte 
apoptosis. Oxygen imbalance develops due to disparity between decreased oxygen supply by low cardiac output and reduced coronary perfusion, and increased demand caused by ventricular wall stress and neurohormonemediated rise of heart rate and contractility. Subsequently, myofibrillar proteins could be also determined in the HF patient without any acute coronary event.

Markers of Myocyte stress (BNP, NT-proBNP, Midregional fragment of proadrenomedullin, ST2). The increasing intracardiac volume and filling pressure result in end-diastolic wall stress, that entails the release of BNP or its precursor NT-pro-BNP directly from the myocardium into the systemic circulation. Both BNP and NT-proBNP are extensively used in the differential diagnosis of dyspnea, and in the management of patients with HF, especially in acute settings. Also, both biomarkers are proven predictors of outcome and valuable parameters in assessing the response to therapy in HF of various etiologies.

\section{Biomarkers beyond clinical utility}

For the clinician, biomarkers from blood can be used for identifying patients with HF, assessing the severity of the disease, stratifying prognosis, and guiding therapy. In addition to that, biomarkers are an interesting subject for research, and several articles on the topic have been published in the Romanian Journal of Laboratory Medicine in recent years.

A review on the role of biomarkers in HF from the point of view of the laboratory specialist is presented by Pop et al. In this review, taking into account the fact that biomarkers are continuously being evaluated in order to bring them closer in clinical practice, different aspects of assessment and clinical implication of the use of biomarkers were regarded. Furthermore, the authors analyse the latest achievements in the methods of qualitative and quantitative assess- ment of biomarkers. Novel techniques, such as the ,omics” (genomics, transcriptmoics, metabolomics) permit a new perspective of the matter of disease pathophysiology. (6)

BNP and NT-proBNP have a well-established role in the diagnosis and prognosis of HF. Measurement of BNP and NT-proBNP is useful in the setting of uncertainty to support the clinical diagnosis of acute decompensated or ambulatory HF. Elevated levels of BNP or NT-proBNP are suggestive of worse clinical outcomes and mortality in HF. NT-proBNP changes to effort are less well studied. The aim of the clinical study performed by Zdrenghea et al. was to compare the new fixed walking test (400mWT) with 6MWT and maximal exercise test by using NT-proBNP levels before and after exercise. In was shown that in both groups with maximal and submaximal exercise, NT-pro-BNP level rises significantly and in the same way. As these biomarkers are released stimuled by two submaximal test, their assessment after stress-testing could be considered as the alternative way of evaluation of excercise tests. (7)

The purpose of another study was to investigate the possible clinical and paraclinical significance of Apelin-13, another strong endogenous inotropic peptide which plays a big role in neutralising the adverse effects of aldosterone and angiotendin 11 in HF patients. It was demonstrated that Apelin-13 negatively correlates with NTpro-BNP. Most of the patients with adverse outcomes had the low level of Apelin-13, especially those presented in acute decompensated HF. (8)

Under mechanical stress, cardiomyocytes and fibroblasts synthetise Galectin- 3 a $\beta$-galactoside-binding lectin member of the galectin family. Therefore, Galectin-3 was hypothesised as a promoter of left ventricular dysfunction. The detailed information about the role of galectin-3 in mediation of inflammation by different pathways is presented in an extensive review by Lupu et al. In this review the clinical utility of the detection of this biomarker is provided. (9) 
Another important tool involved in progression of chronic HF, oxidative stress and inflammation, was investigated by Iliesiu et al. The objective of this study was to evaluate the association between seric uric acid, oxidative stress and inflammation markers in patients with moderate-severe HF reduced left ventricular ejection fraction. It was shown that elevated level of uric acid is correlated with disease severity and in the absence of renal failure may mirror increased xanthine-oxidase activity associated with chronic inflammatory response. (10)

Not only uric acid but also other routine markers (i.e. serum iron concentration) are important in monitoring HF patient severity and outcomes. Due to the supposition that iron deficiency could be an independent risk factor for poor outcomes in HF patients, a Romanian prospective, multicenter, observational study with longitudinal follow up (FERIC-RO) is launched in this issue of the Romanian Journal of Laboratory Medicine. (11) This study will provide evidence about the prevalence and the predictors of iron deficiency, will explore the relationship between alteration in iron homeostasis in patients hospitalized for chronic worsening HF and will bridge knowledge gaps regarding the relationship between in-hospital iron deficiency and post-discharge outcomes.

All of these demonstrate that rapid progress is made in biomarker science. However, unavoidable challenges arise regarding costs or regulatory barriers.

\section{Acknowledgement}

Authors declare no conflict of interest.

\section{References}

1. Ponikowski P, Voors AA, Anker SD, Bueno H, Cleland JG, Coats AJ et al. 2016 ESC Guidelines for the diagnosis and treatment of acute and chronic heart failure. Eur Heart J. 2016;37:2129-200. DOI: $10.1093 /$ eurheartj/ehw128
2. Katz AM, Konstam MA, The Neurohormonal Response in Heart Failure: Functional Signaling in Katz AM, Konstam MA, Geart Failure: Patophysiology, Molecular Biology and Clinical Managemeny, 2th ed, Lippincott Williams \& Wilkins, Philadelphia 2009, pg. 89-133.

3. WHO International Programme on Chemical Safety. Biomarkers in Risk Assessment: Validity and Validation. 2001. Retrieved from http://www.inchem.org/documents/ehc/ehc/ehc222.htm

4. Braunwald E. Biomarkers in Heart Failure. N Engl J Med 2008;358:2148-59. DOI: 10.1056/NEJMra0800239

5. Chow SL, Maisel AS, Anand I, Bozkurt B, de Boer RA, Felker GM, et al. Role of Biomarkers for the Prevention, Assessment, and Management of Heart Failure: A Scientific Statement From the American Heart Association. Circulation. 2017;135(22):e1054-e91. DOI: 10.1161/CIR.0000000000000490

6. Pop C, Crişan GC, Loghin F, Mogoşan CI. Advances in the detection and quantification of candidate and established biomarkers in heart failure. Rev Romana Med Lab. 2013;21(3):255-65. DOI: $10.2478 / \mathrm{rrlm}-2013-0029$

7. Zdrenghea DT, Ilea M, Zdrenghea MD, Sitar-Tăut AV, Pop D. The Effects of Maximal and Submaximal Exercise Testing on NT-proBNP Levels in Patients with Systolic Heart Failure. Rev Romana Med Lab. 2014;22(1):25-33. DOI: 10.2478/rrlm-2014-0008

8. Goidescu CM, Anton FP, Leucuța DC, Mircea PA, Vida-Simiti LA. Serum level of apelin-13 negatively correlated with NT-proBNP in heart failure patients. Rev Romana Med Lab. 2016;24(4):399-412. DOI: 10.1515/rrlm-2016-0035

9. Lupu A, Lupu S, Agoston-Coldea L. Is galectin-3 a promoter of ventricular dysfunction?. Rev Romana Med Lab. 2018;26(1):21-36. DOI: $10.2478 / \mathrm{rrlm}-2018-0001$

10. Iliesiu A, Campeanu A, Marta D, Parvu I, Gheorghe G. Uric Acid, Oxidative Stress and Inflammation in Chronic Heart Failure with Reduced Ejection Fraction. Rev Romana Med Lab. 2015;23(4):397-405. DOI: 10.1515/rrlm-2015-0039

11. Antohi EL, Tatu Chitoiu G, Ambrosy AP, Coman IM, Vinereanu D, Collins SP et al. Rationale and Design of a Prospective, Multicenter, Observational Study Evaluating Iron Deficiency in Patients Hospitalized for Heart Failure (FERIC-RO). Rev Romana Med Lab. 2018;26(3):271-81. DOI:10.2478/rrlm-2018-0022 